Document downloaded from:

http://hdl.handle.net/10251/60826

This paper must be cited as:

Martínez-Román, J.; Pérez Cruz, J.; Pineda Sánchez, M.; Puche Panadero, R.; Roger Folch, J.; Riera Guasp, MV.; Sapena Bañó, Á. (2015). Electrical Machines Laminations Magnetic Properties: A Virtual Instrument Laboratory. IEEE Transactions on Education. 58(1):159-166. doi:10.1109/TE.2014.2348536.

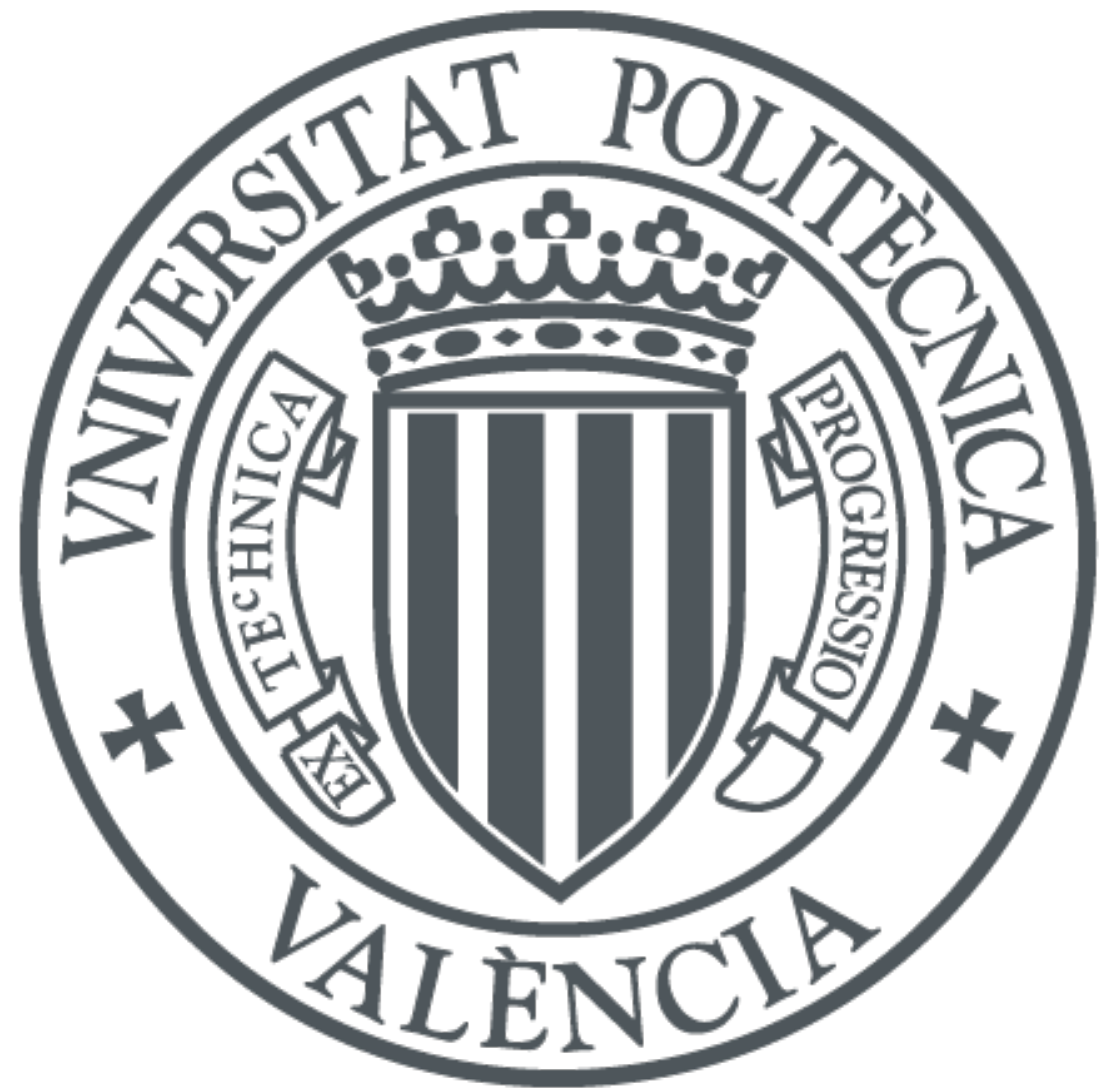

The final publication is available at

Copyright Institute of Electrical and Electronics Engineers (IEEE)

Additional Information

"(C) 2015 IEEE. Personal use of this material is permitted. Permission from IEEE must be obtained for all other uses, in any current or future media, including reprinting/republishing this material for advertising or promotional purposes, creating new collective works, for resale or redistribution to servers or lists, or reuse of any copyrighted component of this work in other works." Upon publication, authors are asked to include either a link to the abstract of the published article in IEEE Xplore®, or the article's Digital Object Identifier (DOI). 


\title{
Electrical Machines Laminations Magnetic Properties: a Virtual Instrument Laboratory
}

\author{
J. Martinez-Roman, J. Perez-Cruz, Member, IEEE, M. Pineda-Sanchez, Member, IEEE, R. Puche-Pa- \\ nadero, Member, IEEE, J. Roger-Folch, Member, IEEE, M. Riera-Guasp, Senior Member, IEEE, and \\ A. Sapena-Baño.
}

\begin{abstract}
Undergraduate courses in electrical machines often include an introduction to their magnetic circuits, and to the various magnetic materials used in their construction and their properties. The students must learn to be able to recognize and compare the permeability, saturation and losses of these magnetic materials, relate each material to its specific properties, and understand the impact of these properties on the major performance metrics of electrical machines. This paper describes a new test equipment setup and lab guide that helps students achieve these learning goals. The test equipment consists of two transformers of grain-oriented and non-grain-oriented electrical steel, transducers, a data acquisition (DAQ) board and a PC-based virtual instrument. The virtual instrument shows voltage, current and core flux time waveforms, the rms voltage versus current curves and, most importantly, the lamination material magnetic cycle. Students' laboratory work was organized into a series of experiments that guide their achievement of these magnetic materials-related abilities. Pre- and post-lab exams assessed student learning, which was shown to have increased significantly. Students' opinions of the relevance, usefulness and motivational effect of the laboratory was also positive.
\end{abstract}

Index Terms - Electrical engineering, electrical engineering education, magnetic circuits, magnetic materials, student experiments, test equipment

\section{INTRODUCTION}

$\mathrm{M}$ agnetic circuits are often an introductory aspect of undergraduate electrical machines courses $[1,2,3,4,5]$. Basic learning objectives in these courses are that students should be able to how the magnetic circuits work, and calculate their main relationships, such as those between core flux and the electrical machine's rated power. Students must also be able to recognize the various lamination categories, in particular grain-oriented electrical steel (GOES) and non-grain-oriented electrical steel (NGOES), and to compare their properties and relate these to their typical uses. Student must be aware that some lamination properties (such as permeability and total specific losses) are directly related to the electrical machine's fundamental performance figures such as efficiency, the no-load current, or the power factor. Traditionally, students have been able, to a very limited extent, to check these facts experimentally with no-load tests at different voltages on typical electrical machines, like transformers and induction machines.

There are significant challenges to teaching electromagnetism and electrical machines because of the use of abstract concepts that are hard to visualize [6], [7], and to the lack of appropriate measurement equipment and test rigs. In the case of electromagnetism, these difficulties are sometimes aggravated by the use of a mathematical approach when actually the students need to visualize the concepts to fully understand them. In recent decades the use of simulations to help visualize those abstract concepts [6], and to simulate the actual electrical machine performance during tests $[8,9,10,11]$ has sometimes been adopted as a means to overcome these difficulties. There are some drawbacks to using simulation tools in electrical engineering courses, however, among them the time needed for students to become familiar with them, or students' perception that these are of limited helpfulness [12]. A mixed virtual instrument approach, proposed here, that blends the test of actual machines of classical experiments with the enhanced data processing and visualization offered by simulations, might profit from their advantages while avoiding their weaknesses.

Virtual instruments (VI) consist of a transducer board, a Digital Acquisition (DAQ) board, a computer and the application specific software (providing DAQ control, acquired data manipulation and handling, and the user interface). In the widespread terminology of DAQ cards and development software manufacturers, the application-specific software is often referred to as the virtual instrument. The VI is connected to actual equipment under test, put under various operating conditions by means of actuators that may or may not be driven by the VI; the equipment's performance is measured, processed and shown to the user by the VI. Vis, which must not be mistaken for simulated systems, have been shown to have many advantages over traditional instruments. Reference [13], as early as 1984, showed the suitability of VIs to help students get a 'real-time' handle on some electrical machine concepts. In [14], efficient data collection and manipulation by means of VIs is shown to help maintain student interest and reduce the time required to perform and evaluate experiments; similar advantages were reported in [15]. VIs' ability to provide user-friendly interaction with the experiment is also underlined in [16]. The modular and reusable nature of Vis, and therefore their suitability for integration into cost-effective systems is underlined in [17]. Addi- 
tional benefits from having students collaborate in VI development is that these then reflect student interests and needs, as described, along with already mentioned advantages, in [18]. With respect to magnetic materials properties, some advantages are also cited in [19], [20].

The curricular changes in European education driven by the Bologna Process [21] and the ensuing curricular redesign in the Escuela Técnica Superior de Ingenieros Industriales, Universitat Politécnica de Valencia (UPV) led to a redesign of the Electrical Machines (EM) course. This course covers magnetic circuits $(20 \%)$, transformers $(40 \%)$ and rotating electrical machines $(40 \%)$, and is taken in their sixth semester by all students in the Industrial Technologies (GITI) and Energy (GIE) engineering degree programs, and by about $25 \%$ of the Industrial Engineering Master's (MII) degree program students; the total annual enrollment is about 500 students. The redesign of the EM course was perceived as an excellent opportunity to upgrade the electrical machines laboratory. The original lab, which focused on changes in magnetic circuit structure and their effect on winding inductance and no-load current, was broadened to include the magnetic properties of various magnetic materials and their impact on key aspects of electrical machine performance.

This paper first briefly introduces, in Section II, the main types of magnetic lamination used in electrical machines, touching on their very different magnetic properties, how these influence key electrical machine performance figures and how they relate to various aspects of the no-load current of a transformer. Section III describes a new VI-based equipment setup designed to help students understand the properties of magnetic laminations and to correlate these with their impact on key transformer operation parameters such as no-load current and core losses. Section IV describes the tests to be performed and the data to be collected to arrive at the main results directly related to the lab's learning objectives. The impact of the lab on students' learning, and their opinion on the relevance, usefulness and motivational effect of the laboratory is detailed in Section V. The main conclusions of are presented in Section VI.

\section{MAGNETIC LAMINATIONS: TYPES, PROPERTIES AND THEIR EFFECT ON SIMPLE MAGNETIC CIRCUITS}

An electrical machine's magnetic core is usually built by stacking electrical steel (ES) sheets, each less than $1 \mathrm{~mm}$ thick, to keep eddy-current losses as low as possible. The steel used generally has about a $4 \%$ silicon content so as to have: a) a thinner magnetic hysteresis loop due to lower coercivity and lower conductivity (lower hysteresis and eddy-current losses) and b) a higher Curie temperature than structural steels (thus allowing for better magnetic properties at higher temperatures). Two different types of steel, distinguished by their grain orientation, show specific magnetic properties that make them suitable for different uses. The first, non-grain-oriented electrical steel (NGOES) has roughly isotropic properties, while grain-oriented electrical steel (GOES) has a considerable higher permeability and lower specific losses when magnetized along the grain. However, if magnetized transversally to the grain, GOES' permeability and specific losses are far worse than those of NGOES. This makes GOES ideal for transformer cores where the magnetic field has a constant direction. In contrast, GOES is rarely used in rotating electrical machines as the magnetic material is often exposed to a rotating magnetic field.

From an electrical engineer's point of view, the main magnetic materials properties are the permeability and specific losses, because they are directly related to fundamental performance figures of the electrical machine. For an electrical machine of given dimensions, the ES permeability is directly related to the magneto-motive force ( $\mathrm{mmf}$ ) required to excite the machine's magnetic core. This mmf accounts for between $30 \%$ (in rotating electrical machines) to $90 \%$ (in transformers) of the total required $\mathrm{mmf}$, the remaining percentage being due to airgaps. In turn, the total mmf is directly related to the machine's no-load current and, through that, to its power factor and to the reactive power consumption. The ES specific losses are directly related to the core losses, amounting to about one third of the machine's total losses. The remaining two thirds are mainly Joule effect losses in the windings and friction losses in rotating machines.

The ES permeability and specific losses can be related to the no-load alternating voltage and current waveforms of a winding coiled around a rectangular magnetic core, just like that of a transformer. The transformer being at no load, the winding sinusoidal voltage is almost equal to the induced emf that, in turn, requires a sinusoidal core flux that must lag the winding voltage by $1 / 4$ period:

$$
\begin{aligned}
& u(t)=\sqrt{2} U \cos (\omega t)=N \frac{d \Phi_{\text {core }}}{d t} \Rightarrow \\
& \Rightarrow \Phi_{\text {core }}=\frac{\sqrt{2} U}{N \omega} \cos (\omega t-\pi / 2)
\end{aligned}
$$

The winding no-load current, necessary to provide the magnetic circuit mmf and to support its losses, can then be split into its magnetizing and iron-loss components. The magnetizing current is in phase with flux pulsation but, due to core saturation, exhibits obvious peaks (in phase with core flux peaks which, in turn, coincide with the zero voltage instant due to the $1 / 4$ period lag) that results in a bell-shaped waveform. The ironloss current is in phase with the winding voltage and, therefore, leads the magnetizing current by a $1 / 4$ period. The result of this phase shift between the two no-load current components appears as a left-peak-right asymmetry of the no-load current semi-periods as in the left part of Fig. 1 (see for example [1], pp. 81-84] or [5], chapter 2.4.2.3]).

The two main differences between NGOES and GOES are the average permeability and the specific losses. These can be observed by comparing the no-load current waveforms of two transformers, identical but for their core ES type, fed by the same sinusoidal voltage. The GOES transformer has a clearly smaller rms no-load current with more symmetric half-periods around the peak current than the NGOES, due to its higher permeability and lower specific losses. However, the differences in permeability and specific losses appear clearly when the instantaneous core flux is plotted against the no-load waveform: GOES has a far steeper and thinner magnetic cycle with lower coercivity than NGOES. This comprehensive magnetic cycles 

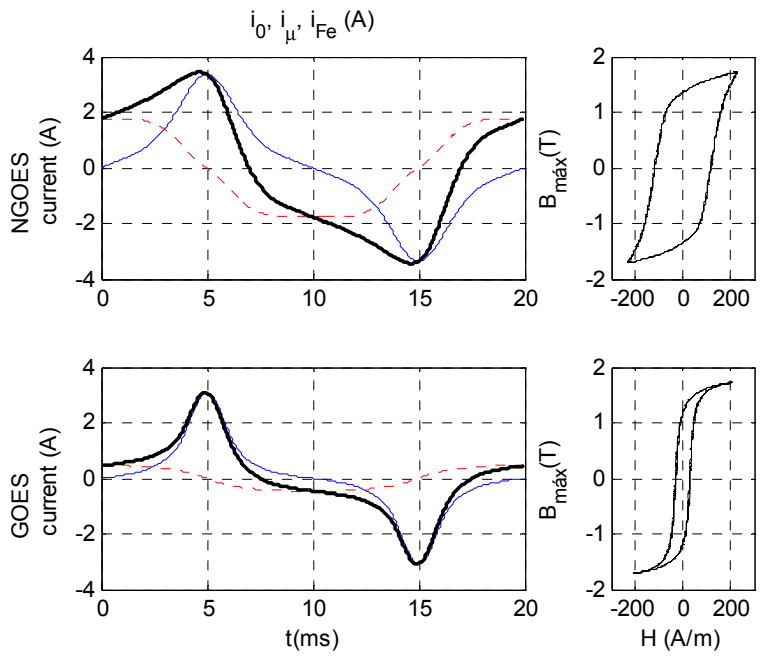

Fig. 1. Left: No load (thick), magnetizing (thin) and iron-loss (dotted) currents. Right: comprehensive magnetic cycle. Top: NGOES. Bottom: GOES.



Fig. 2. EMMATE components diagram: three leg transformer to be tested, tailored transducer board based on Hall effect current transducers and voltage divider/instrumentation amplifier, MC USB-2523 DAQ board and PC-based Virtual Instrument.

\begin{tabular}{|l|c|}
\hline & NGOES \\
\hline Power & $3 \mathrm{kVA}$ \\
\hline Voltage & $380 / 380 \mathrm{~V}$ Yy \\
\hline Current & $4,55 / 4,55 \mathrm{~A}$ \\
\hline Core stack length & $70 \mathrm{~mm}$ \\
\hline Core height & $250 \mathrm{~mm}$ \\
\hline Core width & $250 \mathrm{~mm}$ \\
\hline Window height & $150 \mathrm{~mm}$ \\
\hline Window width & $50 \mathrm{~mm}$ \\
\hline Lamination & M600-50 \\
\hline
\end{tabular}

Table 1. Characteristics of the transformers under test.

(core flux vs. no load current) provide additional information (highly valuable from the core design standpoint) if the axes are properly scaled (as in the right part of Fig. 1): core flux divided by core cross-section to show the core average magnetic induction and no-load current multiplied by the winding turns and divided by the average core length to show the average core magnetic field strength (see eqn. (2)).

\section{TEST EQUIPMENT FOR ELECTRICAL MACHINE'S MAGNETIC CIRCUIT MATERIALS}

To help the student recognize the different properties of NGOES and GOES, and how these affect key performance parameters of electrical machines, an Electrical Machines Magnetic Circuit Materials Test Equipment (EMMATE) was developed. EMMATE has three main components: the transformers, the transducers and DAQ board, and the virtual instrument, see Figs. 2 and 3). The combination of a transducer board tailored to the application, a DAQ board and a specially-designed VI offers the already-described advantages over traditional instruments. The main advantage is its ability to organize and present the measured data in a format that helps the student to identify the specific properties of the different laminations, and to correlate these with the key performance figures of the transformers under test: the no-load current and losses. In addition to the three main components, the system uses a three-phase autotransformer to adjust the various test voltages applied to the transformers, up to $120 \%$ of rated voltage.

\section{A. Transformers}

The transformers under test are two 3kVA, 380/380 Yy three-phase three-leg transformers built with the same dimensions and winding turns, Table 1. Identical but for the lamination type used - one NGOES and one GOES - they were built specifically to help the students compare the effect of the lamination type on machine performance. The power and voltage ratings were selected to work with the existing laboratory mains voltage and autotransformers while being a good compromise between low cost and high-enough rated power. Other transformer ratings can be chosen to suit other laboratories' constraints, as long as the transducer board is adapted appropriately. However, lower rated powers should be avoided, because the transformer parameters would be far away from typical values for power transformers, and work with low-rated power transformers might give a student the false impression that these are "toys', and so neglect security. Although these are threephase, three-leg transformers, only one phase (the left leg) is fed, with all the other windings being kept open except for the secondary winding of the right leg, which is short-circuited. This short circuit forces a very low induced emf and a very low leg flux. In this way the flux lines excited by the left leg winding current can only close themselves through the central leg, clearly simplifying the effective magnetic circuit.

\section{B. Transducers and DAQ Board}

The transducer board was developed to provide high bandwidth and linearity voltage and current signals in three-phase systems, although in this application only one-phase magnitudes are required and measured, Fig. 2. The voltage transduc- 
ers are based on a three-leg 1:100 voltage divider and three instrumentation amplifiers, NI INA128, in differential mode and with a gain of 2, providing an accuracy and linearity better than $0.5 \%$ with a bandwidth of close to $1 \mathrm{MHz}$. The current transducers are two 5A Hall effect transducers, LEM HY-5P, with accuracy and linearity better than $1 \%$ and a bandwidth in the range of $50 \mathrm{kHz}$.

The DAQ board, a Measurement Computing USB-2523, has 8/16 multirange differential/single-ended inputs with a maximum sampling rate of $1 \mathrm{MS} / \mathrm{s}$ muxed and 16-bit resolution. As only two measurements are required for this application, the sampling rate could be as high as $500 \mathrm{kS} / \mathrm{s}$. The board is limited to $50 \mathrm{kS} / \mathrm{s}$ per channel in the virtual instrument to provide adequate time resolution without unnecessary data transmission and calculation load. A USB connection ensures a high throughput, very long acquisition time span, easy installation and high compatibility. Although a simpler, and cheaper, DAQ board would have sufficed for this application, this higher-performance board is also employed to register transients of converter-fed electrical drives and servos used in other labs. This illustrates a further advantage of VI-based labs: cost reduction through component reuse.

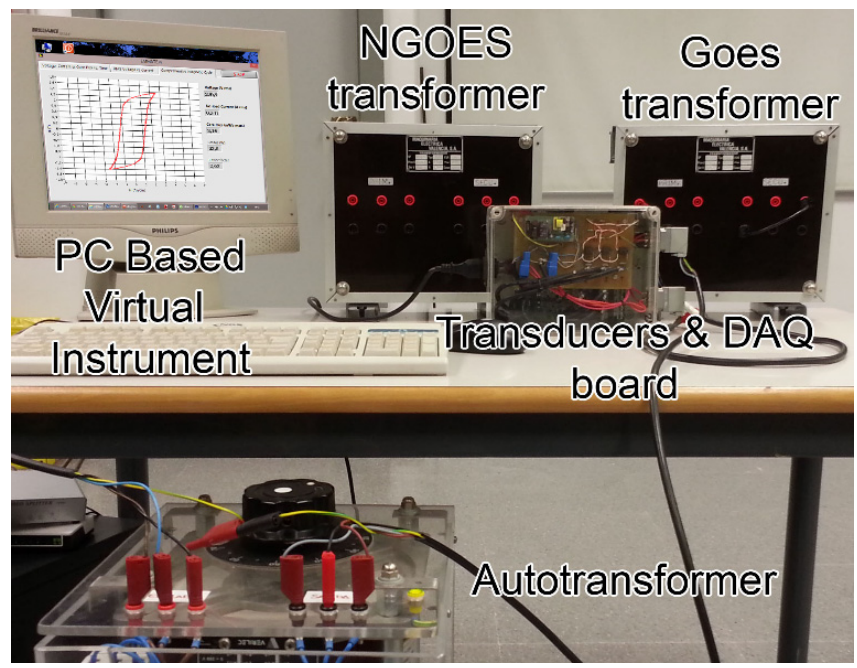

Fig. 3. EMMATE photograph. On the table, right: transformers under test, in front of them: transducers and DAQ board, and left: computer monitor with VI front panel showing the 'live' magnetic cycle.

\section{Virtual Instrument}

The VI manages the DAQ board, and stores, manipulates and presents the test data, and was designed to provide students with the information required to identify the specific properties of the different laminations, and correlate these with two key performance figures of the transformers under test: the no-load current and losses. At start-up, the VI asks the DAQ board for a cyclic data capture of the channels corresponding to the voltage applied and current flowing through the left leg primary winding of the transformer under test. Each data capture extends for the duration of two whole cycles $(40 \mathrm{~ms}$ at $50 \mathrm{~Hz}$ mains frequency) at a sample rate of $100 \mathrm{kS} / \mathrm{s}$. From this measurement the VI calculates the rms values of voltage and current, average electric power and power factor. Assuming no-load operation, and using the known winding turns, the VI estimates the core instantaneous magnetic flux as well as its maximum value. This estimation consists of a numeric voltage wave integration plus a scale change, eqn. (1).

With the known core geometry the VI then calculates the instantaneous core magnetic induction, $\mathrm{B}(\mathrm{t})$, and, assuming negligible airgaps between core sections, the instantaneous value of the average magnetic field strength along the core, $\mathrm{H}(\mathrm{t})$ :

$$
B(t)=\frac{\Phi(t)}{S_{\text {core }}} ; H(t)=\frac{N \cdot i(t)}{l_{\text {core }}}
$$

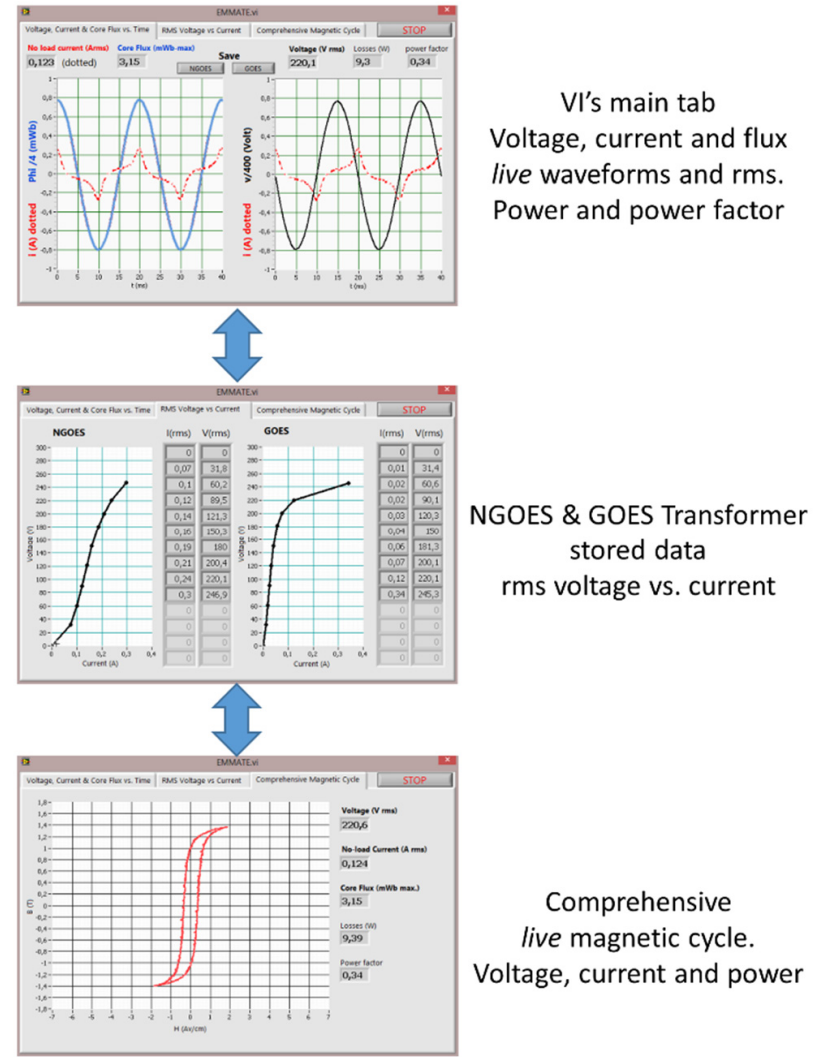

Fig. 4 EMMATE VI user interface tabs: Time waveforms, rms values and power (main tab) - NGOES and GOES transformer stored data rms Voltage vs Current - Comprehensive magnetic cycle.

The user interface is divided into three tabs, Fig. 4, grouping the captured and calculated data. The main tab, Figs. 5 and 6 , shows two time graphs (voltage and current, right, and core flux and current, left) as well as all rms, average or maximum relevant quantities (voltage, current, flux, power and power factor). The information in this main VI tab helps the student recognize: a) the lag between no-load current and voltage, b) the in-phase relationship between core flux and no-load current maxima, c) the direct relationship between voltage and no-load current and transformer core losses. However, this information is especially relevant when the performance figures and no-load current waveforms of the GOES and NGOES transformers, operated at the same voltage, are compared. Then students can quickly correlate the expected higher permeability and lower specific losses of the GOES with its lower rms no-load current, its more symmetrical no-load current waveform and its lower power 
consumption.

This main VI tab also includes buttons to save the actual data shown as a set belonging either to NGOES or the GOES transformer. This data is saved locally (with a filename stating date, time of capture and if it is for the NGOES or GOES transformer) in the computer running the VI. The data is also backed up and made accessible to students in PoliformaT, UPV's elearning platform [22]. A slight delay introduced between consecutive captures introduces a slow sweep effect to the time varying graphs that adds to the 'live' sensation of the actual size change when voltage changes.

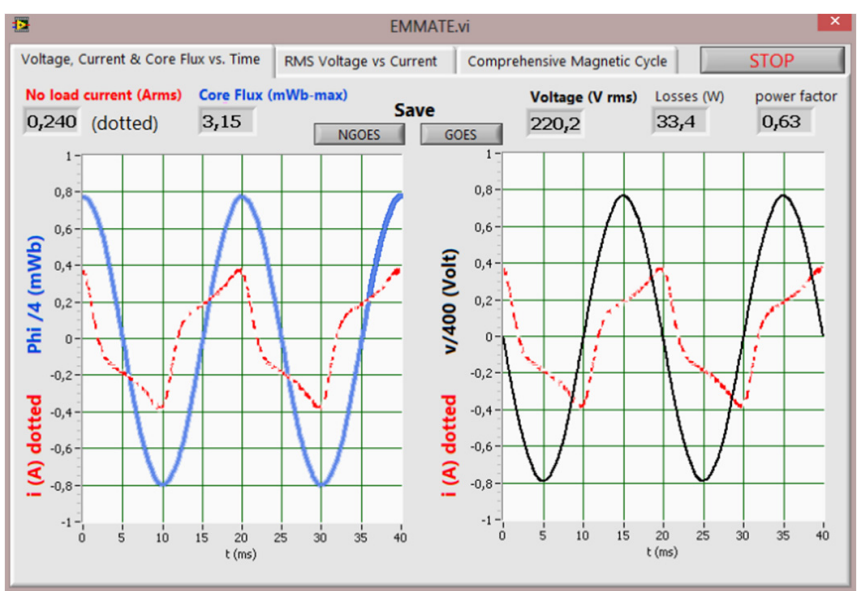

Fig. 5. Virtual Instrument main tab showing core-flux \& current waveforms (left), voltage \& current waveforms (right) as well as rms voltage and current, power and power factor (top). NGOES transformer tested at rated winding voltage.

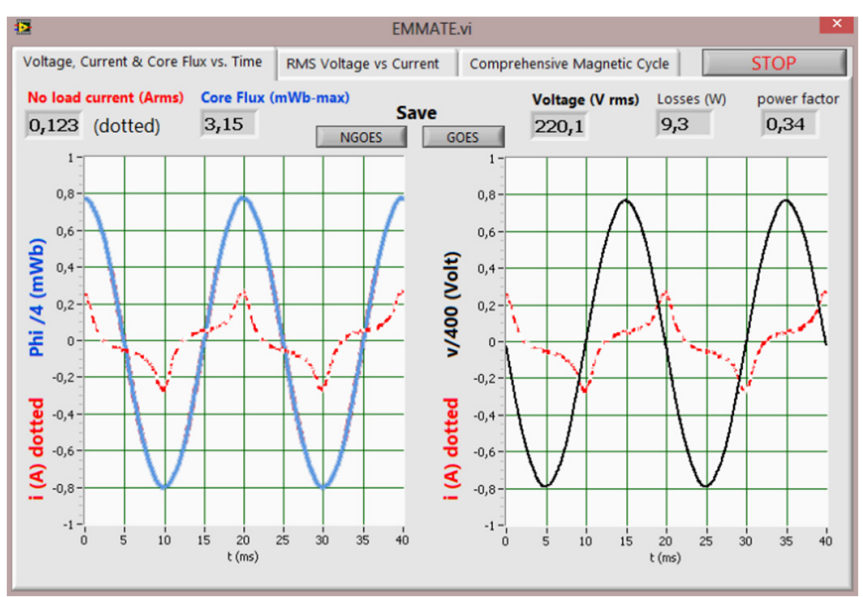

Fig. 6. Same as Fig. 5 but for a GOES transformer tested at rated winding voltage.

The second tab shows the plot of the rms voltages and currents saved during the tests performed in the main tab, Fig. 4 bottom. These two curves are traced with the rms voltage-current pairs stored by clicking the buttons on the main VI tab of the VI; they reproduce the no-load performance curves obtained with traditional instruments. Their inclusion in the VI is mainly to show the user how little information they provide in comparison with the time waveforms, and especially with the comprehensive magnetic cycle. Finally, the third tab shows the core lamination magnetic cycle, Figs. 7 and 8 , by plotting the instantaneous core magnetic induction vs. core magnetic field strength curve according to eqn. (2). This includes, for background, the actual rms voltage and no-load current, as well as the core losses.

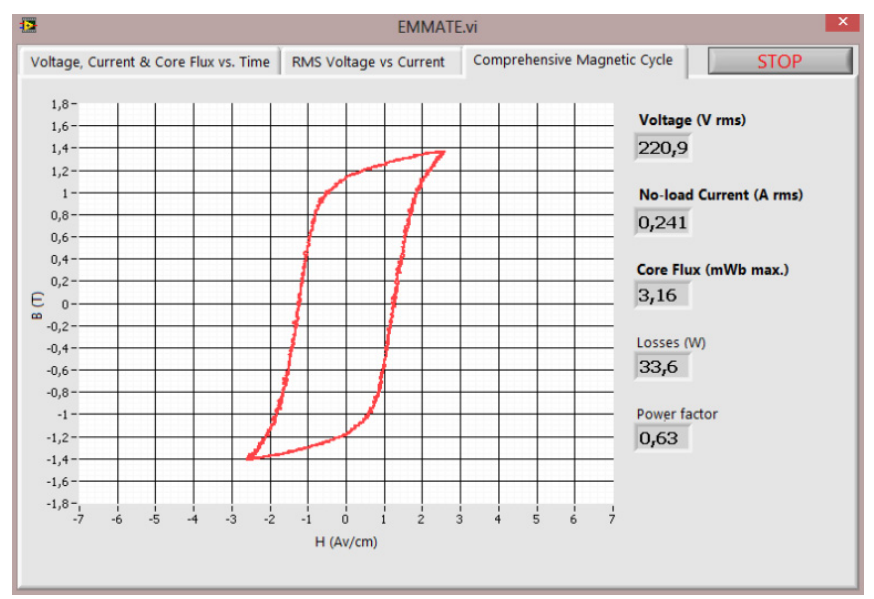

Fig. 7 Comprehensive (hysteresis+eddy current losses) lamination magnetic cycle tab of the VI (NGOES transformer, rated voltage).

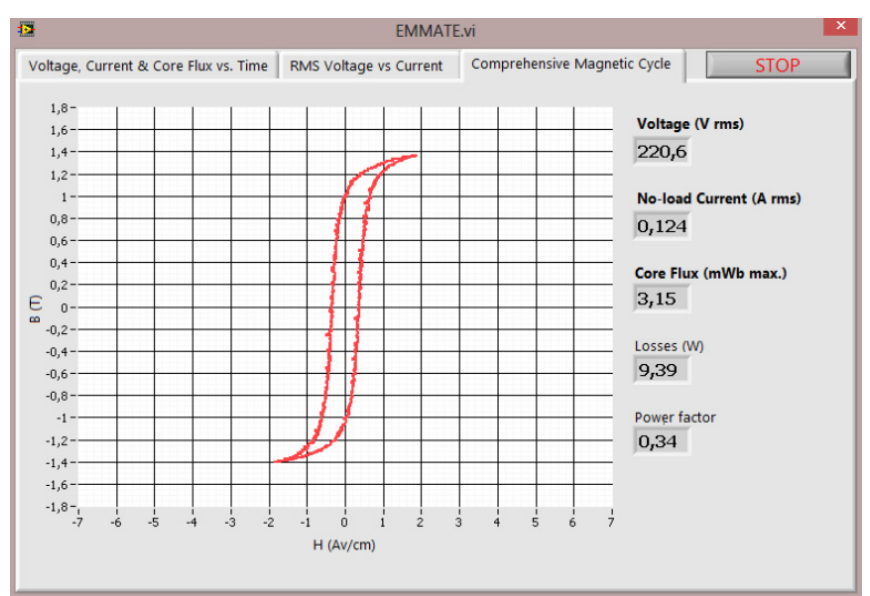

Fig. 8 Comprehensive (hysteresis+eddy current losses) magnetic cycle of the GOES transformer.

It is important to inform the students that this comprehensive magnetic cycle cannot, and does not, separate hysteresis and eddy current losses, as the current measured is the no-load current and includes both loss components. If students are not aware of this fact they can mistake the magnetic cycle shown by the VI with the lamination hysteresis loop; this can be obtained with very low frequency measurements, but not with EMMATE. Other assumptions and sources of error should also be discussed with students, such as the effect of: a) the very small gaps between overlapped laminations on the total $\mathrm{mmf}$ required by the magnetic circuit and on the no-load current, b) neglecting Joule effect winding losses, c) the non-uniform core field distribution.

\section{LABORATORY SESSION GUIDE AND MAIN TEST RESULTS}

The various online graphs and results provided by the VI give 
the teacher and students complete freedom to explore the performance of the magnetic circuit under test. Following a prepared lab procedure, however, guides the user in performing the tests, acquiring data and recognizing the main results, so as to achieve the learning objectives, Fig. 9. The tests are carried out first for the NGOES transformer and then repeated for the GOES transformer. Where comparisons are to be made between the NGOES and the GOES transformers performance, the guide prompts the student to record or collect the necessary data, and make the comparison.

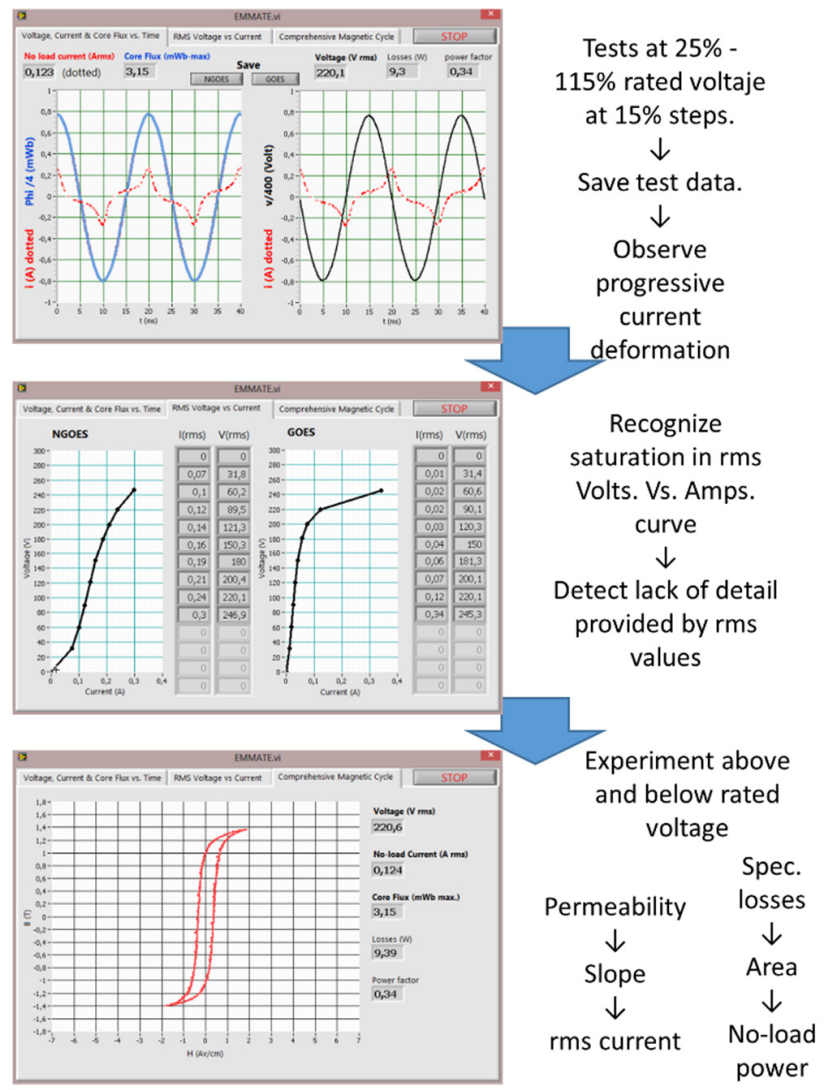

Fig. 9 Virtual Instrument test sequence as described in the lab guide, shown for a GOES transformer. The same test would already have been performed for the NGOES transformer.

The general procedure starts by closing the mains relay with the autotransformer set to $0 \%$ output voltage. The voltage must then be gradually increased to $115 \%$ of the rated voltage, with the student being advised to capture and save data in steps of about $15 \%$ of the rated voltage, using the Save button in the main VI tab. Students' attention is drawn to the gradually increasing effects of saturation and losses on the current waveform, which shows noticeable peaks and asymmetry, while the voltage and core flux waveforms remain apparently sinusoidal. They are also prompted to notice the strong lamination saturation that can be observed even at voltages clearly below the rated voltage. A link with transformer theory is also made by pointing out the $90^{\circ}$ lag between the voltage and core flux waves, as well as the phase coincidence of the core flux and no load current max values.

Once the applied voltage has been investigated across the required range students then look at the second tab with the rms voltage and current graph, Fig. 4 bottom, to find that lamination saturation can be detected just by plotting rms voltage and current. It is important to emphasize that the initial lamination permeability is related to the slope of the rms voltage vs. current curve, but students should also realize the limitations of rms values compared to instantaneous measurements in providing information about lamination losses.

Finally, in the magnetic cycle tab of the VI, Fig. 7, the user is prompted to gradually change the voltage and then observe the evolution of the magnetic cycle. Attention is drawn to the fact that the lamination initial permeability and specific losses are directly related to two geometrical properties of the magnetic cycle: the slope at null magnetic induction and the cycle surface area. Students are prompted to note the values for these two geometrical properties for later comparison with the values to be obtained for the GOES transformer. The general procedure ends by going to the main VI tab, reducing the voltage to $0 \%$ and opening the mains relay, in preparation to repeat the same procedure with the GOES transformer.

The same sequence is carried out for the GOES transformer, taking care to observe those aspects where differences are to be expected, and to compare these with the values recorded for the NGOES transformer. Specifically, the GOES transformer has:

- Very low current, especially at low voltages, both in terms of rms and maximum value.

- Far lower asymmetry of the no load current.

- Smaller no-load losses at rated voltage.

- A steeper slope of the rms voltage vs. current curve.

- A steeper slope of the magnetic cycle for low magnetic induction values.

- A much thinner magnetic cycle and lower coercivity, (seen by comparing Figs. 7 and Fig. 8).

\section{Evaluation}

EMMATE was used during the Spring 2014 semester by UPV's GITI and GIE students, and had previously been tested with the students of earlier degree programs. EMMATE's effectiveness was measured by first, setting a pre- and post-lab exam after the lectures but before the laboratory began, and then again after the laboratory; and, second, a student survey administered after the laboratory. This assessment was conducted only on some of the laboratory sessions, because of the significant time required to take the exams and complete the survey. The pre- and post-exams were taken by 164 students, and 151 answered the survey.

The exams had four multiple-choice or written-answer questions covering the learning objectives, Fig. 10 summarizes the results. Clearly, the laboratory contributed to the student learning: the average grade (Q.Avg) increased by $34 \%$. The increase of the average mark for the individual questions ranged from $11 \%$ to $76 \%$ (the latter being for the question found hardest before the lab). While not all of this increase in average grade can be can be ascribed to EMMATE, it can be said to have had a positive impact on student learning.

The student satisfaction survey had five statements with a 
five-point scale, from 1 (completely agree) to 5 (completely disagree):

S1. This laboratory was useful for your education on electrical machines

S2. This laboratory helped you to understand concepts and enabled you to apply them in the topic being studied

S3. This laboratory increased your motivation towards learning electrical machines

S4. This laboratory provided you with useful experience that might be of service in your future job

S5. The educational materials used in this laboratory were adequate for the tasks to be performed.

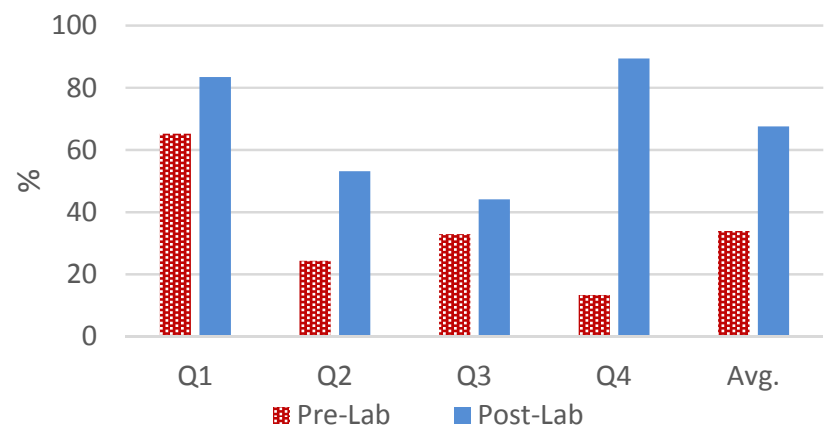

Fig. 10. Pre- and post-laboratory exam results: Average $\%$ grade for all students taking the for all four Q1-Q4 questions and average exam grade



Fig. 11. Student satisfaction survey responses to statements $\mathrm{S} 1$ to S5.

The results of this survey, summarized in Fig. 11, show a good level of student satisfaction with the laboratory, with responses falling mainly between "completely agree" and "agree". The best scores were for statements S1 (usefulness), S2 (aid to understanding and applicability) and S5 (adequacy of the materials), all with over $70 \%$ of responses being between "completely agree" and "agree".

\section{CONCLUSIONS}

Blending modern technologies like DAQ boards and PCbased VIs with traditional electrical machines laboratory equipment has been shown to have many advantages. Modern test equipment and a set of laboratory tests on electrical machines' magnetic materials properties were developed to exploit these advantages, with the aim of helping students identify differ- ences in the properties of various electrical machine core laminations, and their impact on key electrical machine performance parameters.

The test equipment captures voltage and current waveforms during the no-load operation of a transformer. It then processes and presents the actual measured voltage and current waveforms together with the calculated core flux waveform, rms values, power losses and the core magnetic cycle. In this way, through a guided set of tests, the students can directly link the main magnetic properties of the transformer lamination (permeability and specific losses) with basic operation parameters of the transformer (mainly the no-load current and core losses). As the tests are performed on a non-grain oriented lamination transformer and then on a grain oriented lamination one, the students can also perceive the deep impact the change in the lamination magnetic properties has on the transformer performance.

The combination of the guided exercises with the EMMATE test equipment developed resulted in high levels of student satisfaction with the laboratory work, and thus improved student motivation. The laboratory work significantly increased student abilities, as shown by the pre- and post-lab exam results. Both evaluation elements, the survey and the pre and post-lab exams, show that the main objectives set during the laboratory design were achieved.

\section{REFERENCES}

[1] S. J. Chapman, Electric Machinery Fundamentals, 5th ed. McGraw-Hill, 2011.

[2] A. E. Fitzgerald, C. Kingsley, and S. D. Umans, Electric Machinery, 6th ed. McGraw-Hill International Edition, New York, 2003.

[3] S. A. Nasar and L. E. Unnewehr, Electromechanics and electric machines, 2nd ed. Wiley, 1983.

[4] M. G. Say, Alternating current machines, 5th ed. John Wiley \& Sons, 1983.

[5] L. Serrano-Iribarnegaray and J. Martínez-Román, Máquinas Eléctricas. Editorial de la Universitat Politècnica de València, 2013.

[6] V. Pulijala, A. Akula, and A. Syed, "A web-based virtual laboratory for electromagnetic theory," in Technology for Education (T4E), 2013 IEEE Fifth International Conference on, Dec 2013, pp. 13-18.

[7] S. Bentz, "Integration of basic electromagnetism and engineering technology," in Frontiers in Education Conference, 1995. Proceedings., 1995, vol. 2, Nov 1995, pp. 4a5.4-4a5.7 vol.2.

[8] S. Ayasun and C. Nwankpa, "Induction motor tests using matlab/simulink and their integration into undergraduate electric machinery courses," $E d$ ucation, IEEE Transactions on, vol. 48, no. 1, pp. 37-46, Feb 2005.

[9] A. Bentounsi, H. Djeghloud, H. Benalla, T. Birem, and H. Amiar, "Computer-aided teaching using matlab/simulink for enhancing an im course with laboratory tests," Education, IEEE Transactions on, vol. 54, no. 3, pp. 479-491, Aug 2011.

[10] M. Ojaghi, J. Faiz, M. Kazemi, and M. Rezaei, "Performance analysis of saturated induction motors by virtual tests," Education, IEEE Transactions on, vol. 55, no. 3, pp. 370-377, 2012.

[11] A. Syal, K. Gaurav, and T. Moger, "Virtual laboratory platform for enhancing undergraduate level induction motor course using matlab/simulink," in Engineering Education: Innovative Practices and Future Trends (AICERA), 2012 IEEE International Conference on, July 2012, pp. $1-6$.

[12] C. Cañizares and Z. Faur, "Advantages and disadvantages of using various computer tools in electrical engineering courses," Education, IEEE Transactions on, vol. 40, no. 3, pp. 166-171, Aug 1997.

[13] S. Gruber, "A computer-interfaced electrical machines laboratory," Education, IEEE Transactions on, vol. 27, no. 2, pp. 73-79, 1984. 
[14] J. M. Williams, J. L. Cale, N. D. Benavides, J. D. Wooldridge, A. C. Koenig, J. L. Tichenor, and S. D. Pekarek, "Versatile hardware and software tools for educating students in power electronics," Education, IEEE Transactions on, vol. 47, no. 4, pp. 436-445, 2004.

[15] J. M. Jiménez-Martínez, F. Soto, E. d. Jodar, J. A. Villarejo, and J. RocaDorda, "A new approach for teaching power electronics converter experiments," Education, IEEE Transactions on, vol. 48, no. 3, pp. 513-519, 2005

[16] F. Sellschopp and L. Arjona, "An automated system for frequency response analysis with application to an undergraduate laboratory of electrical machines," Education, IEEE Transactions on, vol. 47, no. 1, pp. 5764, 2004.

[17] S. Durovic, "Development of a simple interactive laboratory exercise for teaching the principles of velocity and position estimation," International Journal of Electrical Engineering Education, vol. 50, no. 3, pp. 256-267, 2013.

[18] W. Heath, O. Onel, P. Green, B. Lennox, Z. Gai, Z. He, and M. Rodriguez Liñan, "Developing a student-focused undergraduate laboratory," International Journal of Electrical Engineering Education, vol. 50, no. 3 , pp. 268-278, 2013.

[19] T. Sloane, "Laboratories for an undergraduate course in power electronics," Education, IEEE Transactions on, vol. 38, no. 4, pp. 365-369, Nov 1995.

[20] J. Van't Hof, J. Bain, R. M. White, and J.-G. Zhu, "An undergraduate laboratory in magnetic recording fundamentals," Education, IEEE Transactions on, vol. 44, no. 3, pp. 224-231, Aug 2001.

[21] The European Higher Education Area, Joint Declaration of the European Ministers of Education, Bologna Declaration 1999, 1999, Std. [Online]. Available: $\quad$ http://www.ehea.info/Uploads/Declarations/BOLOGNA_DECLARATION1.pdf

[22] R. Mengod, "Poliformat, the sakai-based on-line campus for upv-history of a success," in 5th Sakai Conference, Vancouver, BC, Canada, vol. 30, 2006.

Javier Martinez-Román was born in 1965 . He received his M.Sc. and Ph.D. degrees, both in electrical engineering from the Universitat Politècnica de Valencia, Spain, in 1993 and 2002. He is an Associate Professor with the Electrical Engineering Department, Universitat Politècnica de Valencia. His primary areas of interest are electrical machines and drives.

Juan Perez-Cruz (M '09) obtained his M.Sc. degree in 1997 and his Ph.D. degree in 2006 from the Universitat Politecnica de Valencia, both in electrical engineering. From 1970 to 1992 he worked in the electrical industry in the field of industrial systems maintenance and automation. In 1992 he joined the Universitat Politecnica de Valencia and is currently an Associate Professor of electrical installations and machines. His research interests focus on induction motor diagnostics, numerical modelling, and automation.

Manuel Pineda-Sanchez (M '02) received his M.Sc.degree in 1985 and his $\mathrm{Ph} . \mathrm{D}$. degree in 2004 from the Universitat Politecnica de Valencia, both in electrical engineering. Currently he is an Associate Professor in the Department of Electrical Engineering of the Universitat Politecnica de Valencia. His research interests include electrical machines and drives, induction motor diagnostics, and numerical simulation of electromagnetic fields.

Ruben Puche-Panadero (M '09) received his M.Sc. degree in Automatic and Electronic Engineering in 2003, and his Ph.D. degree in electrical engineering in 2008, both from the Universitat Politecnica de Valencia. He joined the Universitat Politecnica de Valencia in 2006 and he is currently an Associate Professor of Control of Electrical Machines. His research interests focus on induction motor diagnosis, numerical modelling of electrical machines, and advanced automation processes and electrical installations.

Jose Roger-Folch (M '03) received the M.Sc. degree from the Universidad Politecnica de Cataluña, Barcelona, Spain, in 1970, and the Ph.D. degree from the Universitat Politecnica de Valencia, Valencia, Spain, in 1980, both in electrical engineering. From 1971 to 1978 , he worked in the electrical industry as a project engineer. Since 1978, he has been with the Department of Electrical Engineering, Universitat Politecnica de Valencia, where he is currently a Professor of Electrical Installations and Machines. His main research areas are numerical methods applied to the design and maintenance of electrical machines and equipment.

Martin Riera-Guasp (M '94-SM '12) received the M.Sc. degree in industrial engineering and the Ph.D. degree in electrical engineering from the Universitat Politecnica de Valencia (Spain) in1981 and 1987, respectively. Currently he is an Associate Professor in the Department of Electrical Engineering of the Universitat Politecnica de Valencia. His research interests include condition monitoring of electrical machines and electrical systems efficiency.

Angel Sapena-Baño obtained his M.Sc. degree in 2008 and his $\mathrm{Ph} . \mathrm{D}$. degree in 2014 from the Universitat Politecnica de Valencia, both in electrical engineering. Since 2008 he has worked as a Researcher in the Institute for Energy Engineering of Universitat Politecnica de Valencia. His research interests focus on induction motor diagnostics and maintenance based on condition monitoring, numerical modeling of electrical machines, and advanced automation processes and electrical installations. 